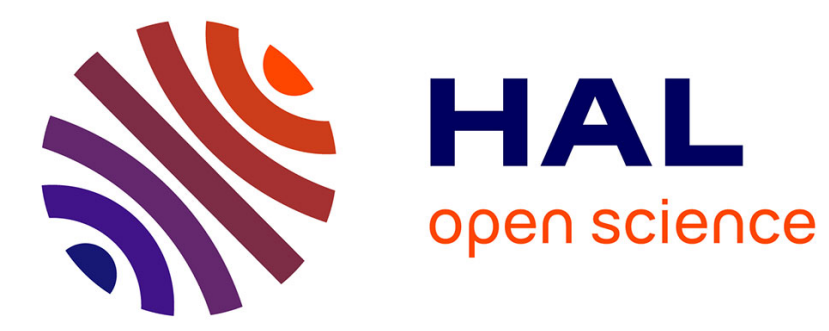

\title{
Aider un parent âgé dépendant. Configurations d'aide et interactions dans les fratries en France
}

Quitterie Roquebert, Roméo Fontaine, Agnès Gramain

\section{To cite this version:}

Quitterie Roquebert, Roméo Fontaine, Agnès Gramain. Aider un parent âgé dépendant. Configurations d'aide et interactions dans les fratries en France. Population (édition française), 2018, 73 (2), pp.323-350. 10.3917/popu.1802.0323 . hal-02367727

\section{HAL Id: hal-02367727 \\ https://hal.science/hal-02367727}

Submitted on 26 Nov 2019

HAL is a multi-disciplinary open access archive for the deposit and dissemination of scientific research documents, whether they are published or not. The documents may come from teaching and research institutions in France or abroad, or from public or private research centers.
L'archive ouverte pluridisciplinaire HAL, est destinée au dépôt et à la diffusion de documents scientifiques de niveau recherche, publiés ou non, émanant des établissements d'enseignement et de recherche français ou étrangers, des laboratoires publics ou privés. 


\title{
AIDER UN PARENT ÂGÉ DÉPENDANT. CONFIGURATIONS D'AIDE ET INTERACTIONS DANS LES FRATRIES EN FRANCE
}

\author{
Quitterie Roquebert, Roméo Fontaine et Agnès Gramain
}

Institut national d'études démographiques | « Population »

2018/2 Vol. 73 | pages 323 à 350

ISSN 0032-4663

ISBN 9782733220306

Article disponible en ligne à l'adresse :

https://www.cairn.info/revue-population-2018-2-page-323.htm

Distribution électronique Cairn.info pour Institut national d'études démographiques.

(C) Institut national d'études démographiques. Tous droits réservés pour tous pays.

La reproduction ou représentation de cet article, notamment par photocopie, n'est autorisée que dans les limites des conditions générales d'utilisation du site ou, le cas échéant, des conditions générales de la licence souscrite par votre établissement. Toute autre reproduction ou représentation, en tout ou partie, sous quelque forme et de quelque manière que ce soit, est interdite sauf accord préalable et écrit de l'éditeur, en dehors des cas prévus par la législation en vigueur en France. Il est précisé que son stockage dans une base de données est également interdit. 


\title{
Aider un parent âgé dépendant. Configurations d'aide et interactions dans les fratries en France
}

\begin{abstract}
Avec le vieillissement démographique, la question de la prise en charge des personnes âgées dépendantes devient un enjeu de société majeur. L'État français a développé des politiques en ce sens, mais la famille et les enfants assument également une grande partie de ce travail de care. Lorsqu'une personne est dépendante, le nombre de ses enfants et leur sexe jouent-ils sur l'aide dont elle peut bénéficier? S'il est connu que les filles sont plus souvent aidantes que les fils, que se passe-t-il quand la fratrie n'est pas mixte? Observe-t-on des comportements différenciés des aînés et des cadets ? À partir des données de l'enquête Handicap-Santé, Quitterie ROQUEBERT, Roméo FONTAINE et Agnès GRAMAIN mettent en évidence des asymétries de genre et de rang concernant la fratrie dans l'aide apportée par les enfants, qui diffèrent aussi selon le type d'aide considérée.
\end{abstract}

L'aide informelle est aujourd'hui une ressource essentielle en matière d'aide aux personnes âgées dépendantes ${ }^{(1)}$. Cette aide non rémunérée fournie par l'entourage est à la fois plus fréquente, plus intense et plus diversifiée que celle fournie par les aidants formels (professionnels et rémunérés). D’après l'enquête française Handicap-Santé (Insee-Drees, 2008-2009), parmi les 3,6 millions de personnes de 60 ans ou plus recevant une aide régulière à domicile, $80 \%$ déclarent être aidées par un proche, tandis que 50 \% déclarent avoir recours à de l'aide professionnelle (Soullier et Weber, 2011). Le temps d'aide médian apporté par les aidants informels atteint 1 h40 par jour contre 0 h35 pour les aidants professionnels. Les aidants informels interviennent en moyenne dans

(1) Le terme de " perte d'autonomie » est souvent utilisé pour parler de la dépendance des personnes âgées. Nous choisissons ici d'utiliser le terme de « dépendance » pour insister sur l'état de dépendance plutôt que sur le processus de perte d'autonomie.

* Paris School of Economics, Université Paris 1.

** Institut national d'études démographiques, Paris.

*** BETA, Université de Lorraine.

Correspondance : Quitterie Roquebert, Paris School of Economics - bureau R3-68, 48 Boulevard Jourdan, 75014 Paris, courriel : quitterie.roquebert@univ-parisl.fr 
la réalisation de quatre tâches de la vie quotidienne, contre deux pour les aidants professionnels (Fontaine, 2011). Ce constat n'est pas propre à la France. Dans les pays européens, malgré la diversité des systèmes de protection sociale et des normes d'accompagnement, l'aide informelle joue un rôle prépondérant dans la prise en charge des personnes âgées dépendantes (Colombo et al., 2011).

L'importance du rôle joué par l'entourage des personnes âgées dépendantes invite non seulement à décrire les configurations d'aide informelle utilisées, mais aussi à comprendre le processus sous-jacent à leur formation. Ce processus revêt d'autant plus d'intérêt que les évolutions démographiques vont affecter les configurations familiales, par la réduction de la descendance finale et l'augmentation attendue du nombre de personnes âgées dépendantes vivant en couple. La mobilisation effective des enfants et le partage de l'aide dans la fratrie peuvent dépendre de déterminants dits «structurels», qui influencent la décision d'aide d'un enfant indépendamment de la décision des autres membres de la famille. C'est le cas si l'aide est affectée par des caractéristiques individuelles comme la situation familiale ou le statut d'emploi, par des variables parentales, comme le genre ou le niveau de dépendance du parent, ou par des variables de fratrie, comme sa composition par sexe $e^{(2)}$. Mais le fait d'aider est aussi éventuellement lié à celle des autres aidants potentiels. Ces « interactions endogènes » peuvent modifier sensiblement l'impact attendu des politiques publiques et des évolutions démographiques : par exemple, si les enfants s'organisent pour qu'au moins l'un d'eux soit impliqué, alors la réduction de la taille des fratries aura peu d'effet, aussi bien sur l'aide apportée par l'aidant principal que sur l'existence d'aide informelle pour le parent dépendant.

Ce travail propose d'éclairer plusieurs questions sur les configurations d'aide familiale à un parent âgé : comment l'implication ${ }^{(3)}$ des enfants s'ajuste-t-elle aux caractéristiques familiales? Comment, au sein des fratries de 2 enfants, s'articulent les décisions de chacun? Existe-t-il des logiques d'implication différentes selon le rang des enfants dans la fratrie? Enfin, la formation des configurations d'aide repose-t-elle sur les mêmes principes selon le type d'aide considéré?

Nous proposons d'abord de décrire, à partir des données françaises de l'enquête Handicap-Santé, les configurations d'aide mises en place au sein des fratries confrontées à la dépendance d'un parent âgé, telles qu'elles sont déclarées par celui-ci. Nous questionnons en particulier la sensibilité des configurations d'aide filiale aux caractéristiques familiales (nombre d'enfants et présence d'un conjoint auprès du parent). L'analyse se concentre ensuite sur les déterminants de la décision d'aider des enfants ayant un seul frère ou sœur, en tenant compte de la présence possible d'interactions endogènes en plus des déterminants structurels. Isoler les interactions endogènes permet de mettre

(2) Considérer la composition de la fratrie permet de se demander si être une femme a le même effet sur le comportement d'aide lorsque l'autre enfant est une femme ou un homme.

(3) Les termes « implication » ou « mobilisation » désignent le fait d'aider ou non, indépendamment de l'intensité, de la fréquence ou de la nature de l'aide apportée. 
en évidence l'interdépendance des comportements d'aide. De manière originale, l'effet de ces déterminants est différencié selon le rang dans la fratrie; on compare ces effets selon deux définitions de l'aide, l'une recouvrant plusieurs types d'aide (aide aux tâches de la vie quotidienne, aide financière ou soutien moral) et l'autre ciblée sur l'aide aux tâches de la vie quotidienne.

\section{Vers la modélisation des interactions au sein de la fratrie}

Les premiers travaux sur la formation des configurations d'aide se sont attachés à décrire la désignation de « l'aidant principal », en supposant qu'un seul enfant est désigné comme aidant dans la fratrie. Ils visaient à mettre en lumière l'effet des caractéristiques personnelles de l'enfant et du parent sur le comportement d'aide de l'enfant (Engers et Stern, 2002; Hiedemann et Stern, 1999; Pezzin et Steinberg Schone, 1999; Sloan et al., 1997; Stern, 1994). Cette approche est toutefois remise en cause par les données empiriques, puisque l'aide est fréquemment fournie par plusieurs enfants simultanément. En Europe, en 2004, parmi les personnes âgées seules et dépendantes avec 2 enfants, 1 sur 5 recevait de l'aide de ses 2 enfants (Fontaine et al., 2009). On retrouve une proportion semblable d'enfants aidants dans les fratries de 2 enfants en France en 2008, selon l'enquête Handicap-Santé (voir infra). Analyser la formation des configurations d'aide familiale implique donc de ne pas se limiter à un seul aidant, fût-il principal.

Dès lors que l'on envisage la possibilité d'aides simultanées, on peut se demander si les comportements des enfants sont indépendants : la décision d'aide d'un enfant est-elle influencée par le comportement d'aide des autres membres de sa fratrie? Cette question a conduit certains auteurs vers des modèles inspirés de la théorie des jeux, qui prennent en compte les anticipations que forment les agents sur les décisions d'aide des autres enfants (Byrne et al., 2009; Checkovich et Stern, 2002; Engers et Stern, 2002; Hiedemann et Stern, 1999; Pezzin et al., 2007). En cas d'interdépendance des comportements, il peut exister plusieurs formes de lien : l'aide apportée par un enfant se faitelle plutôt en substitution ou en complément de celle apportée par les autres? Lanalyse sociologique montre que l'on peut observer aussi bien des mobilisations d'enfants « en équipe » que des processus de retrait de la part de certains enfants lorsque d'autres sont aidants (Pennec, 2007). Il est aussi possible que les interactions ne soient pas homogènes : tous les enfants réagissent-ils de la même manière à l'implication des autres, quel que soit leur rang dans la fratrie? Autoriser une telle asymétrie est une caractéristique originale de la modélisation utilisée par Fontaine et al. (2009). Leurs résultats, obtenus à partir de l'enquête Survey on Health, Ageing and Retirement in Europe (SHARE), font effectivement apparaître des comportements différenciés entre aînés et cadets dans le cas particulier des fratries de 2 enfants dont le parent âgé dépendant vit sans conjoint. 
Repérer empiriquement l'existence d'interactions endogènes suppose de pouvoir les isoler des autres facteurs pouvant affecter les comportements d'aide. D’après les travaux de Manski (2000), trois mécanismes peuvent expliquer l'association des comportements au sein d'un groupe. Le premier mécanisme correspond aux « interactions endogènes » et renvoie à l'interdépendance directe des comportements individuels au sein d'un groupe. Mais l'association des comportements peut aussi provenir d'« interactions contextuelles », qui désignent l'effet direct des caractéristiques d'un individu sur le comportement d'un autre membre du groupe : par exemple, un enfant dont le frère est inactif peut considérer que ce frère a du temps à consacrer à leur parent et choisir de ne pas s'impliquer dans l'aide, même si, de fait, son frère n'apporte aucune aide. Enfin, les « effets corrélés », troisième mécanisme, sont liés à l'existence d'un contexte commun aux individus d'un même groupe, qui peut être imparfaitement observé dans les enquêtes. La spécificité du contexte familial partagé peut influencer simultanément la décision de chacun des enfants : ce contexte recouvre le besoin d'aide du parent commun ou la présence de son conjoint, mais aussi les normes familiales, qui peuvent être partagées entre enfants mais restent souvent inobservées.

Isoler les interactions endogènes des autres sources de corrélation est essentiel lorsque l'on souhaite analyser les logiques de partage de l'aide au sein de la famille. La présence d'interactions endogènes a une conséquence majeure : tout facteur modifiant l'implication d'un membre de la famille modifiera également, de manière indirecte, l'aide apportée par les autres membres de la famille. Dans l'évaluation des politiques publiques, l'existence d'interactions endogènes implique donc de tenir compte des effets indirects des politiques d'aide aux aidants. Une politique ciblant les aidants en emploi affectera, de manière indirecte, les aidants potentiels à la retraite s'ils interagissent avec un frère ou une sœur en emploi. Dans un contexte de vieillissement de la population, isoler l'effet des interactions endogènes est également essentiel pour les projections des ressources en aide informelle : au sein d'une famille, la moindre mobilisation des uns pourrait être compensée par une mobilisation accrue des autres, remettant ainsi en cause le quasi-consensus sur la diminution programmée des ressources en aide informelle. Comme le souligne Manski, l'identification statistique des interactions endogènes est toutefois délicate : elle suppose des données riches et des modélisations spécifiques.

\section{L'étude de l'aide filiale aux personnes âgées dépendantes dans l'enquête Handicap-Santé}

Mise en œuvre en France en 2008-2009 par la Drees et l'Insee, l'enquête Handicap-Santé offre les données nécessaires à l'analyse des interactions endogènes. Elle porte sur les problèmes de santé et les difficultés rencontrées 
dans la vie quotidienne par la population française. Nous utilisons ici le volet Ménages de l'enquête (HSM), centré sur les personnes vivant en ménage ordinaire. Nous étudions l'aide reçue par les personnes âgées dépendantes ayant au moins 1 enfant, en discutant les définitions de l'aide mobilisées dans l'enquête.

\section{L'échantillon de l'étude}

Notre analyse porte sur les configurations familiales d'aide apportée à des personnes âgées dépendantes vivant à domicile et ayant au moins 1 enfant, cas dans lequel l'aide informelle joue un rôle majeur et où différentes configurations d'aide peuvent émerger. Pour chaque enquêté, outre un ensemble de caractéristiques individuelles, on connaît les aides formelles et informelles éventuellement reçues et leurs principales modalités. Les enquêtés sont également interrogés sur leur famille; on possède un ensemble de données sociodémographiques pour chacun de leurs enfants (sexe, âge, situation familiale, situation d'emploi, cohabitation avec le parent ou proximité géographique) ainsi que les modalités de son implication (aidant ou non, type d'aide, fréquence, volume horaire). Disposer de ces informations pour chaque enfant, aidant ou non, est essentiel pour traiter des déterminants de la décision d'aide.

Étudier les personnes âgées à domicile implique une sélection parmi l'ensemble de la population âgée dépendante : si elles sont majoritaires en nombre, les personnes dépendantes restant à domicile sont plus souvent des femmes, moins dépendantes et avec un réseau familial plus important que les personnes en établissement d'hébergement (Désesquelles et Brouard, 2003; Duée et Rebillard, 2006). Notre analyse des configurations d'aide doit donc être comprise comme une analyse conditionnelle au choix de résider en domicile ordinaire.

Nous considérons comme " âgé » tout individu de 60 ans ou plus. Ce seuil a été choisi en référence à la législation française : à partir de 60 ans, un individu devient éligible aux allocations pour les personnes âgées ${ }^{(4)}$ (Tenand, 2016). Nous considérons comme « dépendant » un individu qui déclare avoir besoin de l'aide d'un tiers pour réaliser au moins une activité de la vie quotidienne, qu'il s'agisse d'une activité dite «élémentaire » (ADL, Activities of daily living) - manger et boire, s'habiller, aller aux toilettes, se coucher et se lever, s'assoir et se lever - ou « instrumentale » (IADL, Instrumental activities of daily living) - préparer ses repas seul, faire des courses, faire le ménage, prendre des

(4) Ce seuil de 60 ans demande de faire la distinction entre les personnes âgées dépendantes et les personnes handicapées vieillissantes. Dans le cas de parents handicapés vieillissants, il est probable que les configurations d'aide familiale soient antérieures à l'entrée dans la vieillesse et possèdent des caractéristiques propres à la prise en charge du handicap. Avec les informations disponibles sur les allocations perçues par les personnes âgées dépendantes, 13 individus de plus de 60 ans percevant des allocations spécifiques au handicap ont été identifiés et supprimés de l'échantillon des personnes âgées dépendantes. 
médicaments, aller dehors, prendre les transports en commun, utiliser un ordinateur ou un téléphone. Avec cette définition relativement large, allant au-delà des soins personnels ou de l'aide aux tâches domestiques, 10,6 \% des personnes âgées de 60 ans ou plus (2226 individus dans l'échantillon) étaient dépendantes en $2008^{(5)}$.

Le tableau 1 présente la répartition des personnes âgées dépendantes selon leur situation conjugale et leur nombre d'enfants vivants. La description des configurations d'aide au sein des fratries menées dans la section suivante est restreinte aux 1883 personnes âgées dépendantes ayant au moins 1 enfant. Lanalyse des déterminants structurels et des interactions menée par la suite sera quant à elle restreinte aux personnes âgées dépendantes ayant 2 enfants : en excluant les fratries avec jumeaux, l'échantillon final est composé de 479 fratries ${ }^{(6)}$.

Tableau 1. Répartition des personnes âgées dépendantes selon le statut conjugal et le nombre d'enfants

\begin{tabular}{|c|c|c|c|c|c|c|}
\hline \multirow[b]{2}{*}{$\begin{array}{l}\text { Nombre d'enfants } \\
\text { vivants de la } \\
\text { personne âgée } \\
\text { dépendante }\end{array}$} & \multicolumn{2}{|c|}{ Ensemble } & \multicolumn{2}{|c|}{ Sans conjoint } & \multicolumn{2}{|c|}{ Avec conjoint } \\
\hline & $\begin{array}{c}\text { Nombre } \\
\text { d'observations } \\
\text { dans } \\
\text { l'échantillon }\end{array}$ & $\begin{array}{c}\text { Fréquence } \\
\text { dans } \\
\text { la population } \\
(\%)\end{array}$ & $\begin{array}{c}\text { Nombre } \\
\text { d'observations } \\
\text { dans } \\
\text { l'échantillon }\end{array}$ & $\begin{array}{c}\text { Fréquence } \\
\text { dans } \\
\text { la population } \\
(\%)\end{array}$ & $\begin{array}{c}\text { Nombre } \\
\text { d'observations } \\
\text { dans } \\
\text { l'échantillon }\end{array}$ & $\begin{array}{c}\text { Fréquence } \\
\text { dans } \\
\text { la population } \\
(\%)\end{array}$ \\
\hline Aucun enfant & 307 & 14,9 & 218 & 19,1 & 89 & 9,3 \\
\hline 1 enfant & 425 & 20,0 & 237 & 21,5 & 188 & 18,1 \\
\hline 2 enfants & 492 & 24,8 & 241 & 22,5 & 251 & 27,6 \\
\hline 3 enfants & 351 & 16,1 & 173 & 14,9 & 178 & 17,8 \\
\hline 4 enfants et + & 615 & 24,2 & 327 & 22,0 & 288 & 27,2 \\
\hline Ensemble & 2190 & 100 & 1196 & 100 & 994 & 100 \\
\hline \multicolumn{7}{|c|}{$\begin{array}{l}\text { Notes : Fréquences pondérées. } \\
\text { Champ : } 2190 \text { personnes âgées dépendantes vivant en ménage ordinaire, sans enfant mineur. } \\
\text { Source : Enquête Handicap-Santé, volet Ménages (Insee-Drees, 2008). }\end{array}$} \\
\hline
\end{tabular}

\section{Définition des enfants aidants}

Dans l'enquête, l'aide informelle correspond à une aide régulière liée à la dépendance. Elle est déclarée par les enquêtés lorsqu'ils répondent à la question suivante : «Y a-t-il des personnes (famille, amis...) non professionnelles, qui vous aident régulièrement pour accomplir certaines tâches de la vie quotidienne ou qui vous aident financièrement ou matériellement, ou bien encore qui vous apportent un soutien moral en raison d'un problème de santé ou d'un handicap, y compris les personnes qui vivent avec vous? » Si c'est le cas, l'enquêté liste 10 aidants informels : nous considérons comme aidant un enfant inclus dans

(5) $23,8 \%$ de ces individus déclarent avoir besoin de l'aide d'un tiers pour faire au moins l'une des activités ADL. Parmi les individus restants, 79,1 \% ont au moins deux limitations IADL.

(6) Parmi les 492 personnes âgées dépendantes ayant 2 enfants majeurs, 13 ont des jumeaux : ces fratries sont exclues de l'analyse selon le rang. 
cette liste $^{(7)}$. Notre définition de l'aide repose donc sur la déclaration du parent. Cette mesure n'est pas neutre. Elle comporte en particulier des risques d'invisibilité (si, par exemple, le parent considère que l'aide des enfants va de soi et ne la mentionne pas) ou de sur-déclaration (si, par exemple, il souhaite apparaître comme bien entouré par ses enfants) liés aux caractéristiques de la personne enquêtée et de son entourage (Beliard et al., 2012). En outre, cette mesure ne dit rien du caractère plus ou moins contraint de la décision d'aide ni de l'ampleur ou de la nature de l'aide.

Certains enfants cohabitant avec leur parent n'ont pas été déclarés comme aidants ${ }^{(8)}$. Nous supposons que la cohabitation mène de fait à une aide, au moins pour les tâches de la vie quotidienne, et classons donc comme aidants tous les enfants cohabitant avec leur parent ${ }^{(9)}$. Les enfants cohabitant avec leur parent sans être déclarés aidants représentent $10 \%$ des enfants que nous considérons comme aidants ${ }^{(10)}$.

La définition retenue dans l'enquête recouvre par ailleurs des modalités d'aide variées : elle inclut non seulement l'aide aux tâches de la vie quotidienne (aide à la toilette, habillage, tâches ménagères), mais aussi l'aide matérielle ou financière et le soutien moral. Cette diversité de l'aide fait écho au champ large des activités retenues pour définir la dépendance. Le questionnaire permet de distinguer ces différentes aides souvent concomitantes parmi les enfants déclarés aidants ${ }^{(1)}$ : près de $85 \%$ apportent au moins un soutien moral, $59 \%$ une aide dans les tâches de la vie quotidienne et $18 \%$ une aide financière ou matérielle (figure 1). L'aide financière ou matérielle des enfants est rarement apportée sans un autre type d'aide : son inclusion ne modifie donc qu'à la marge la définition large de l'aide retenue. Considérer le soutien moral comme une modalité d'aide a potentiellement plus d'impact, puisque pour un tiers des enfants qui sont déclarés aidants, l'aide se fait sous cette forme uniquement.

Les résultats présentés s'appuient dans un premier temps sur la définition large de l'aide incluant au moins l'un des trois types d'aide. Cependant, comme les enjeux de coordination entre aidants potentiels sont probablement plus forts pour l'aide dans les tâches de la vie quotidienne, l'analyse des interactions endogènes est aussi conduite avec une définition restreinte aux enfants déclarés aidants pour ce type d'aide particulier.

(7) Nous avons supprimé les cas (3 observations) où tous les enfants ne sont pas cités dans la liste d'aidants et où l'individu déclare par ailleurs avoir plus de 10 aidants informels (non listés). Il est alors possible que l'aide de l'un des enfants ne soit pas observée.

(8) Dans l'ensemble des fratries ayant un parent âgé dépendant, 7 \% des enfants d'un parent âgé cohabitent avec leur(s) parent(s) et $4 \%$ des enfants cohabitent sans être déclarés aidants. Dans les fratries de 2 enfants, $10 \%$ des enfants cohabitent avec leur parent et $3 \%$ cohabitent sans être déclarés aidants (avec des fréquences similaires pour aînés et cadets).

(9) Ce choix nous a amenés à supprimer de notre étude les familles où l'un des enfants est encore mineur (1,6\% des familles avec un parent âgé dépendant et 1,2\% des fratries de 2 enfants avec un parent âgé dépendant), pour lesquels le rapport entre aide et cohabitation est ambigu.

(10) 157 enfants dans l'ensemble des enfants et 27 enfants appartenant à une fratrie de 2 enfants.

(11) À l'exclusion des enfants cohabitants. 
Figure 1. Modalités de l'aide apportée par les enfants aidants d'un parent âgé dépendant

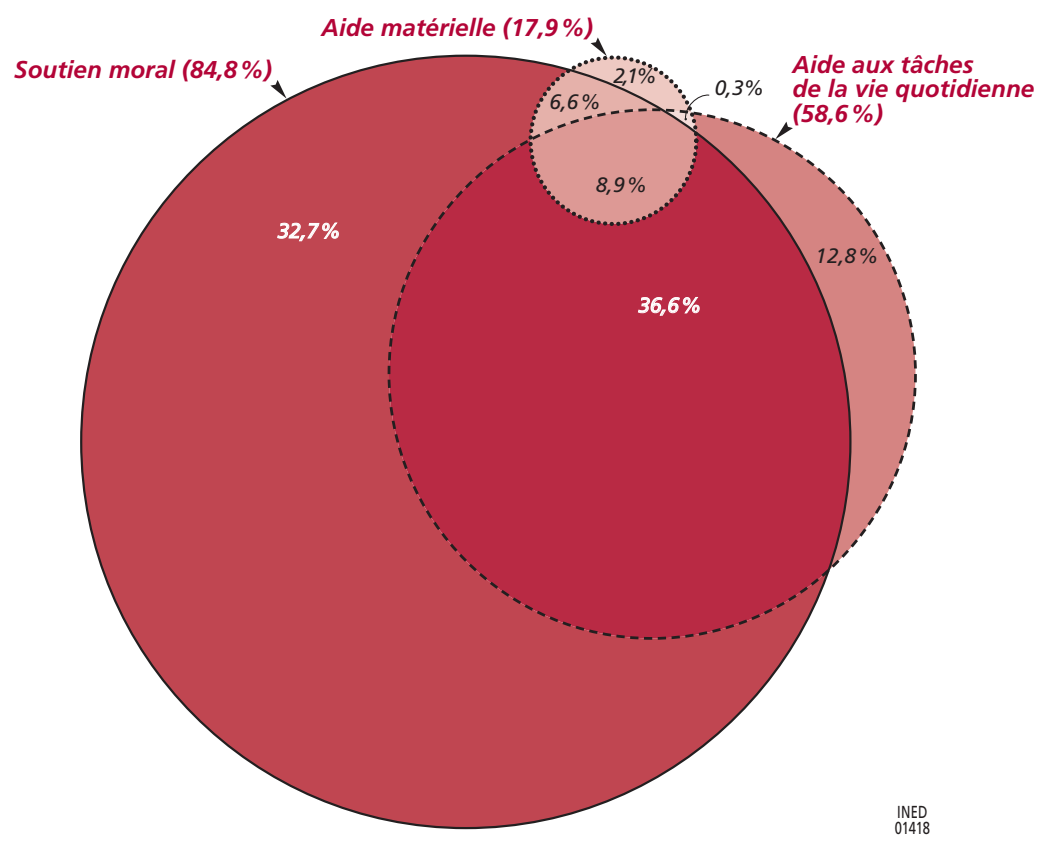

Lecture : $12,8 \%$ des enfants aidants d'un parent âgé dépendant apportent exclusivement une aide aux tâches de la vie quotidienne. Au total, 58,6 \% (12,8\%+36,6\% + 0,3\%+8,9\%) des enfants aidants apportent au moins une aide aux tâches de la vie quotidienne.

Notes : Fréquences pondérées. Un enfant est considéré ici comme aidant uniquement s'il est déclaré aidant par le parent. Les 157 enfants de l'échantillon cohabitant avec leur parent et non déclarés aidants ne sont pas inclus.

Champ : 1341 enfants de parents âgés et dépendants, déclarés aidants par leur parent.

Source : Enquête Handicap-Santé, volet Ménages (Insee-Drees, 2008).

\section{Quelles configurations d'aide au sein des fratries?}

La fréquence de l'aide peut être saisie à l'échelle des enfants, par la proportion d'enfants aidants (tableau 2) et à l'échelle des parents, par la proportion de parents aidés par au moins 1 enfant (tableau 3). Comparer ces fréquences selon la situation conjugale du parent et la taille de la fratrie fait apparaître certaines caractéristiques des configurations d'aide filiale dans le cas français.

De manière attendue, l'aide filiale est plus marquée lorsque le parent est seul, qu'on se place à l'échelle des enfants ou à celle des parents. La proportion d'enfants aidants (tableau 2) est jusqu'à 2 fois plus faible lorsque le parent vit en couple, quelle que soit la taille de la fratrie : parmi les enfants uniques dont le parent dépendant est sans conjoint, plus de 1 sur 2 est déclaré aidant, mais la proportion n'est plus que de 1 sur 4 lorsque le parent vit en couple; on retrouve le même schéma parmi les enfants ayant des frères et sœurs. Quant 
Tableau 2. Répartition des enfants d'un parent âgé dépendant selon la taille de la fratrie et la déclaration d'aide (tous types d'aide)

\begin{tabular}{|c|c|c|c|c|}
\hline $\begin{array}{l}\text { Nombre d'enfants } \\
\text { vivants de la personne } \\
\text { âgée dépendante }\end{array}$ & $\begin{array}{c}\text { Nombre } \\
\text { d'observations dans } \\
\text { l'échantillon (enfant) }\end{array}$ & $\begin{array}{l}\text { Nombre d'enfants } \\
\text { aidants ou cohabitants } \\
\text { dans l'échantillon }\end{array}$ & $\begin{array}{l}\text { Proportion } \\
\text { d'enfants } \\
\text { aidants (\%) }\end{array}$ & $\begin{array}{l}\text { Parmi les enfants } \\
\text { aidants, part des } \\
\text { aidants seuls (\%) }\end{array}$ \\
\hline \multicolumn{5}{|c|}{ Parent vivant sans conjoint et ayant } \\
\hline 1 enfant & 237 & 148 & 56,0 & 100 \\
\hline 2 enfants & 482 & 208 & 38,5 & 42,0 \\
\hline 3 enfants & 519 & 162 & 21,1 & 38,4 \\
\hline 4 enfants et + & 1844 & 451 & 26,1 & 26,7 \\
\hline Ensemble & 3082 & 969 & 30,4 & 44,8 \\
\hline \multicolumn{5}{|c|}{ Parent vivant avec un conjoint et ayant } \\
\hline 1 enfant & 188 & 49 & 26,4 & 100 \\
\hline 2 enfants & 502 & 104 & 15,0 & 48,9 \\
\hline 3 enfants & 534 & 106 & 17,9 & 31,9 \\
\hline 4 enfants et + & 1634 & 270 & 15,1 & 34,3 \\
\hline Ensemble & 2858 & 529 & 16,4 & 43,4 \\
\hline \multicolumn{5}{|c|}{$\begin{array}{l}\text { Lecture : Dans les fratries de } 2 \text { enfants où le parent est âgé, dépendant et sans conjoint, 38,5 \% des enfants } \\
\text { sont aidants. } 42 \% \text { d'entre eux sont les seuls aidants de la fratrie } \\
\text { Notes : fréquences pondérées. Un enfant est considéré comme aidant s'il est déclaré aidant par le parent (tous } \\
\text { types d'aide) ou cohabitant. } \\
\text { Champ : } 5940 \text { enfants majeurs ayant un parent âgé et dépendant qui vit en ménage ordinaire. } \\
\text { Source : Enquête Handicap-Santé, volet Ménages (Insee-Drees, 2008). }\end{array}$} \\
\hline
\end{tabular}

Tableau 3. Part des parents âgés dépendants aidés par au moins 1 enfant

\begin{tabular}{|c|c|c|c|}
\hline $\begin{array}{l}\text { Nombre d'enfants } \\
\text { vivants de la personne } \\
\text { âgée dépendante }\end{array}$ & $\begin{array}{l}\text { Nombre d'observations } \\
\text { dans l'échantillon (parent) }\end{array}$ & $\begin{array}{l}\text { Proportion de parents aidés } \\
\text { par au moins } 1 \text { enfant (\%) }\end{array}$ & $\begin{array}{l}\text { Parmi les parents aidés, } \\
\text { part des parents aidés } \\
\text { par } 1 \text { seul enfant }(\%)\end{array}$ \\
\hline \multicolumn{4}{|c|}{ Parent vivant sans conjoint et ayant } \\
\hline 1 enfant & 237 & 52,6 & 100 \\
\hline 2 enfants & 241 & 51,2 & 63,4 \\
\hline 3 enfants & 173 & 37,8 & 58,9 \\
\hline 4 enfants et + & 327 & 67,0 & 56,0 \\
\hline Ensemble & 978 & 53,4 & 69,9 \\
\hline \multicolumn{4}{|c|}{ Parent vivant avec un conjoint et ayant } \\
\hline 1 enfant & 188 & 24,7 & 100 \\
\hline 2 enfants & 251 & 19,1 & 62,9 \\
\hline 3 enfants & 178 & 22,7 & 42,7 \\
\hline 4 enfants et + & 288 & 41,5 & 67,1 \\
\hline Ensemble & 905 & 27,6 & 68,2 \\
\hline \multicolumn{4}{|c|}{$\begin{array}{l}\text { Lecture : } 51,2 \% \text { des personnes âgées dépendantes sans conjoint ayant } 2 \text { enfants reçoivent de l'aide de la part } \\
\text { d'au moins } 1 \text { enfant. Parmi elles, 63,4 \% reçoivent de l'aide de la part de } 1 \text { seul enfant. } \\
\text { Notes : fréquences pondérées. Un enfant est considéré comme aidant s'il est déclaré aidant par le parent (tous } \\
\text { types d'aide) ou cohabitant. } \\
\text { Champ : } 1883 \text { personnes âgées dépendantes vivant en ménage ordinaire et ayant au moins } 1 \text { enfant, sans } \\
\text { enfant mineur. } \\
\text { Source : Enquête Handicap-Santé, volet Ménages (Insee-Drees, 2008). }\end{array}$} \\
\hline
\end{tabular}

à la proportion de parents déclarant recevoir de l'aide d'au moins 1 de leurs enfants (tableau 3), elle est de $27,6 \%$ pour ceux qui sont en couple contre $53,4 \%$ pour ceux qui vivent sans conjoint. Le statut conjugal module également 
la relation entre la taille de la fratrie et l'aide des enfants. Lorsque le parent vit sans conjoint, la proportion d'enfants aidants décroît avec la taille de la fratrie (tableau 2), passant de 1 sur 2 parmi les enfants uniques à 38,5\% dans les familles de 2 enfants, et environ 1 sur 4 pour les familles plus nombreuses. Lorsque le parent vit en couple, la proportion d'enfants aidants dans les fratries de plusieurs enfants est assez stable quel que soit le nombre de frères et sœurs (environ 1 enfant sur 6), et légèrement plus faible que dans les familles avec enfant unique (1 sur 4).

En revanche, la plus forte implication des enfants en l'absence de conjoint semble se faire sans augmentation de la fréquence relative des situations d'aide multiple : en moyenne, 44,8 \% des enfants aidants sont le seul membre de la fratrie à être impliqué lorsque le parent est seul contre 43,4\% lorsqu'il est en couple (tableau 2). De même, parmi les parents qui déclarent être aidés par au moins 1 enfant (tableau 3), la proportion de ceux qui ne déclarent qu'un seul enfant aidant est de $69,9 \%$ lorsqu'ils vivent seuls et de $68,2 \%$ en présence d'un conjoint.

Dans les fratries de plusieurs enfants, la mise en regard des chiffres calculés à l'échelle des enfants et à celle des parents suggère l'existence d'interactions. En effet, la proportion de parents aidés par au moins 1 enfant est toujours inférieure à ce que l'on devrait observer si les déclarations concernant les différents enfants d'une même fratrie étaient indépendantes ${ }^{(12)}$. Que le parent vive seul ou en couple, avoir 2 ou 3 enfants plutôt qu'un seul ne permet pas d'accroître particulièrement la probabilité de recevoir de l'aide.

On peut se demander si les régularités mises en évidence concernent de la même manière tous les enfants de la fratrie selon leur rang (tableau 4). Dans les fratries de 2 enfants, les cadets s'impliquent significativement plus souvent que les aînés dans la prise en charge de leur parent âgé dépendant. Ces différences de comportement s'appuient en partie sur des différences dans les caractéristiques individuelles selon le rang, comme le suggère le tableau 5, ou par un effet différencié selon le rang de caractéristiques données. La plus grande mobilisation des cadets traduit de fait leur plus grande tendance à s'impliquer seuls. Ce résultat conduit à s'interroger sur l'existence d'interactions différentes dans la fratrie selon le rang : est-ce parce qu'aînés et cadets réagissent différemment au comportement d'aide de leur frère ou sœur que les cadets aident plus souvent seuls que les aînés? Ces différences montrent l'importance de distinguer, au moins dans les fratries de 2 enfants, le rang de naissance pour l'étude des déterminants de l'aide. Dans les fratries de 3 enfants, la probabilité d'être impliqué (en général ou seul) augmente également avec le rang, mais de manière moins marqués et non significative.

(12) Ainsi, dans les fratries de 2 enfants dont le parent vit seul, 38,5\% des enfants sont déclarés aidants (tableau 2). Si la déclaration pour l'aîné était indépendante de celle pour le cadet, au moins 1 des enfants serait déclaré aidant dans $62,2 \%$ des fratries $(0,385+0,385-0,3852)$. Dans le souséchantillon correspondant, c'est le cas de seulement 51,2\% des fratries (différence significative avec un risque d'erreur inférieur à $5 \%$ ). 
En travaillant sur les fratries de 2 enfants, nous proposons d'étudier les trois sources potentielles de différences dans l'implication des enfants selon leur rang, à savoir les différences de caractéristiques individuelles, l'impact différent de ces caractéristiques et des caractéristiques familiales sur la décision d'aide, et enfin les différences dans l'ajustement de l'un des enfants au comportement de l'autre.

Tableau 4. Proportion d'enfants aidants selon leur rang dans la fratrie

\begin{tabular}{|l|c|c|c|c|c|c|c|}
\hline & \multicolumn{3}{|c|}{ Fratries de 2 enfants } & \multicolumn{4}{c|}{ Fratries de 3 enfants } \\
\cline { 2 - 7 } & Aîné & Cadet $\begin{array}{c}\text { Test d'indépendance } \\
\text { du Chi' } \\
\text { (probabilité critique) }\end{array}$ & Aîné & Cadet & Benjamin & $\begin{array}{c}\text { Test d'indépendance } \\
\text { du Chi }^{2} \\
\text { (probabilité critique) }\end{array}$ \\
\hline $\begin{array}{l}\text { Proportion } \\
\text { des enfants aidants } \\
\text { (\%) }\end{array}$ & 25,1 & 30,1 & 0,09 & 18,0 & 17,4 & 21,6 & 0,31 \\
$\begin{array}{l}\text { Proportion } \\
\text { des enfants aidants } \\
\text { seuls (\%) }\end{array}$ & 9,4 & 14,4 & 0,09 & 5,4 & 6,2 & 8,3 & 0,55 \\
\hline $\begin{array}{l}\text { Nombre } \\
\text { d'observations }\end{array}$ & 479 & 479 & - & 335 & 335 & 335 & - \\
\hline
\end{tabular}

Lecture : 25,1 \% des aînés d'une fratrie de 2 enfants ayant un parent âgé dépendant sont aidants. 9,4 \% des aînés d'une fratrie de 2 enfants ayant un parent âgé dépendant aident sans l'aide de l'autre enfant de la fratrie (donc 15,7 \% des aînés aident avec leur cadet).

Notes : Fréquences pondérées. Lorsque la valeur de la probabilité critique est inférieure à 0,10 , la différence entre les proportions est significative au seuil de $10 \%$. Un enfant est considéré comme aidant s'il est déclaré aidant par le parent (tous types d'aide) ou cohabitant.

Champ : 1963 enfants majeurs appartenant à des fratries de 2 ou 3 enfants, ayant un parent âgé et dépendant vivant en ménage ordinaire.

Source : Enquête Handicap-Santé, volet Ménages (Insee-Drees, 2008).

Tableau 5. Caractéristiques sociodémographiques des enfants d'un parent âgé et dépendant (fratries de 2 enfants)

\begin{tabular}{|c|c|c|c|}
\hline & Aînés & Cadets & $\begin{array}{c}\text { Probabilité critique } \\
\text { associée au test d'égalité } \\
\text { des moyennes }\end{array}$ \\
\hline Âge $(a n s)^{(a)}$ & 51,6 & 46,7 & 0,00 \\
\hline Femme (\%) & 52,2 & 53,4 & 0,70 \\
\hline En couple (\%) & 71,8 & 68,7 & 0,29 \\
\hline A des enfants (\%) & 80,4 & 75,2 & 0,05 \\
\hline Nombre d'enfants ${ }^{(b)}$ & 2,2 & 2,0 & 0,01 \\
\hline $\begin{array}{l}\text { Actif (occupé ou chômeur) } \\
\text { sur le marché du travail (\%) }\end{array}$ & 67,4 & 75,8 & 0,00 \\
\hline Cohabite avec son parent (\%) & 9,4 & 10,0 & 0,74 \\
\hline Habite hors de la région de son parent (\%) & 28,0 & 22,1 & 0,04 \\
\hline \multicolumn{4}{|c|}{$\begin{array}{l}\text { (a) La moyenne d'âge est calculée sur les enfants pour lesquels le parent a répondu (469 aînés, } 474 \text { cadets). } \\
\text { (b) Parmi ceux ayant des enfants ( } 385 \text { aînés, } 360 \text { cadets). } \\
\text { Notes : moyennes et fréquences non pondérées. Lorsque la valeur de la probabilité critique est inférieure à 0,10, } \\
\text { la différence entre les moyennes est significative au seuil de } 10 \% \text {. } \\
\text { Champ : } 479 \text { aînés et } 479 \text { cadets majeurs d'un parent âgé et dépendant ayant } 2 \text { enfants. } \\
\text { Source : Enquête Handicap-Santé, volet Ménages (Insee-Drees, 2008). }\end{array}$} \\
\hline
\end{tabular}




\section{Expliquer les configurations familiales dans les fratries de 2 enfants}

L'analyse statistique visant à isoler la présence d'interactions endogènes est menée dans le cas spécifique des fratries de 2 enfants. Outre le fait que les différences de comportement selon le rang sont marquées dans ces fratries, ce cadre permet d'éviter d'avoir recours aux hypothèses de formalisation restrictives et difficilement testables qui seraient nécessaires pour fixer un mode d'agrégation des comportements des membres de fratries de plus de 2 enfants. Pour une fratrie de 2 enfants, le comportement des autres se résume, mécaniquement, au comportement d'un seul individu. Les fratries de 2 enfants correspondent par ailleurs à la taille de fratrie la plus fréquente dans les années à venir : selon l'enquête Famille et logements (Insee-Ined, 2011), 34 \% des hommes et 31 \% des femmes nés entre 1931 et 1935 étant parent ont eu 2 enfants; pour les générations nées 30 ans plus tard, ces proportions sont respectivement égales à 46 \% et $45 \%$ (Masson, 2013).

\section{Identifier les interactions : approche méthodologique}

Analyser dans quelle mesure l'implication d'un enfant dépend de l'implication de son frère ou de sa sœur nécessite de s'appuyer sur un modèle statistique tenant compte simultanément du comportement d'aide déclaré des 2 enfants d'une même fratrie. Quatre configurations d'aide sont possibles : aucun enfant n'est aidant, seul l'aîné est aidant, seul le cadet est aidant et les 2 enfants sont aidants.

Pour isoler les interactions endogènes des autres déterminants de l'implication dans l'aide, notre modélisation statistique ${ }^{(13)}$ repose sur l'hypothèse que les configurations d'aide familiale observées présentent les caractéristiques d'un équilibre de Nash : nous supposons qu'au sein d'une fratrie, chaque enfant adopte le comportement qui lui semble le meilleur compte tenu du comportement de son frère ou de sa sœur, mais également de ses préférences et du contexte dans lequel elles s'expriment. Les configurations observées sont donc supposées "stables », au sens où aucun des deux enfants ne veut, à ce stade du processus, changer son comportement étant donné le comportement de l'autre.

Le principal intérêt de cette approche est de faire dépendre la probabilité d'implication de chaque enfant directement de l'implication de son frère ou de sa sœur, après contrôle des déterminants structurels (caractéristiques

(13) Ce modèle a déjà été utilisé par Fontaine et al. (2009) pour étudier les configurations familiales autour d'un parent âgé, seul et dépendant, à partir des données de l'enquête SHARE. Nous présentons dans cet article les principales caractéristiques du modèle. Le lecteur souhaitant une présentation plus formalisée pourra se référer à Fontaine et al. (2009), qui discutent en particulier les enjeux liés à l'incomplétude du modèle (Tamer, 2003). Contrairement à ces auteurs, nous adoptons ici une règle de sélection exogène selon laquelle, en cas d'absence d'équilibre dans une fratrie donnée, chacune des quatre configurations d'aide a une probabilité d'être observée égale à sa fréquence dans l'échantillon. 
individuelles de l'enfant, du parent et de la fratrie). Il est donc possible de tester la présence, et le cas échéant, l'ampleur des interactions endogènes. Ce modèle permet également la différenciation des comportements selon le rang dans la fratrie : les effets estimés sont supposés différents pour les aînés et pour les cadets.

L'implication des enfants dépend de caractéristiques dont certaines peuvent être inobservées et corrélées au sein d'une même fratrie. Nous faisons donc l'hypothèse que les termes aléatoires qui résument ces caractéristiques inobservées sont distribués selon une loi normale bivariée, et estimons le modèle par la méthode du maximum de vraisemblance.

\section{Choix des déterminants structurels}

Nous supposons que la probabilité qu'un enfant soit aidant dépend du comportement d'aide de son frère ou de sa sœur, mais également de ses caractéristiques individuelles, de celles du parent et de la fratrie (description de l'échantillon dans le tableau annexe A.1).

En plus de l'âge de l'enfant ${ }^{(14)}$, nous avons pris en compte ses situations familiale et professionnelle susceptibles d'affecter sa disponibilité (Le BihanYouinou et Martin, 2006; Peyrache et Ogg, 2017). Les enfants des aidants nécessitant de l'attention et du temps, ils peuvent être considérés comme une destination concurrente dans les décisions d'allocation du temps. L'effet du statut conjugal est a priori plus ambigu : être en couple peut diminuer la disponibilité de l'aidant ou permettre le report de l'aide apportée au parent âgé sur le conjoint, c'est-à-dire le bel-enfant (effet négatif attendu). Il peut aussi y avoir un partage des tâches au sein du couple qui libère du temps pour que l'enfant soit aidant. La variable d'emploi distingue les actifs (occupés ou chômeurs) des autres situations (retraité, étudiant, au foyer ou autre $\left.{ }^{(15)}\right)$. Nous supposons que les logiques d'allocation du temps sont différenciées et que le coût d'opportunité de l'aide est plus important pour les actifs. Nous avons également isolé la modalité où le parent n'a pas su renseigner le statut d'emploi de l'enfant. Il est d'autant plus important de raisonner à caractéristiques individuelles données que nos définitions de l'aide incluent les enfants cohabitant avec leur(s) parent(s), dont les profils familiaux et professionnels sont spécifiques (Ogg et al., 2015).

Pour les caractéristiques sociodémographiques du parent, en plus de l'âge, nous avons étudié l'effet du sexe pouvant affecter le niveau et la nature de l'aide. En effet, les femmes déclarent plus souvent recevoir une aide régulière (Bonnet et al., 2013) et l'aide qu'elles reçoivent est plus souvent d'ordre financier et

(14) Dans 5 fratries de notre échantillon, le parent n'a pas su donner l'âge de l'aîné et du cadet. Dans 5 autres, il n'a pas su déclarer l'âge de l'aîné uniquement. Nous avons traité ces valeurs manquantes en les mettant respectivement à la moyenne de l'âge des aînés et de l'âge des cadets.

(15) La modalité « autre » renvoie potentiellement à une situation où l'enfant est déclaré handicapé par rapport au marché du travail. Cette modalité est rare dans notre échantillon (13 aînés et 21 cadets). 
matériel, tandis que les hommes déclarent plus fréquemment recevoir une aide pour les tâches de la vie quotidienne (Soullier, 2012; Soullier et Weber, 2011). Bien que tous les parents étudiés soient dépendants, nous avons distingué un niveau de limitation plus sévère en isolant les individus qui déclarent avoir besoin de l'aide d'un tiers pour au moins l'une des activités élémentaires de la vie quotidienne (ADL). La littérature ayant montré l'importance du conjoint comme aidant principal, plaçant les enfants en seconde ligne (Fontaine et al., 2007; Weber, 2011) - ce que souligne aussi notre description des configurations familiales-, nous avons inclus le statut conjugal du parent dans les déterminants de l'aide. Nous avons aussi testé l'effet du niveau d'études du parent, selon qu'il détient ou pas le baccalauréat, et celui du revenu mensuel, en isolant ceux qui se trouvent dans le dernier quartile de notre échantillon (revenu mensuel au moins égal à $1800 €$ ). Nous supposons que ces variables, corrélées à la position des individus dans l'espace social, affectent l'aide fournie par les enfants en modulant le recours à l'aide professionnelle et les normes familiales de prise en charge (Weber et al., 2014).

Nous avons enfin étudié les variables ayant trait aux caractéristiques de la fratrie. Le statut d'emploi de l'autre membre de la fratrie a été inclus dans les déterminants, en utilisant la distinction entre actifs et les autres situations. $\mathrm{Au}$ lieu d'inclure individuellement le sexe des enfants comme déterminant de l'implication, nous avons inclus la composition sexuée de la fratrie ${ }^{(16)}$. Rarement mobilisée dans la littérature, cette variable permet de tester l'existence d'interactions contextuelles dépendantes du genre. Si l'on sait que les femmes sont plus systématiquement aidantes (Bonnet et al., 2013; Membrado, 2013), on peut se demander dans quelle mesure être une femme affecte différemment le comportement d'aide suivant que l'autre enfant est un homme ou une femme. Enfin, nous avons inclus une variable dichotomique indiquant si la différence d'âge entre l'aîné et le cadet est supérieure à 10 ans.

Notre estimation utilise donc la quasi-intégralité des informations sur les enfants disponibles dans l'enquête. Deux variables n'ont pas été directement mobilisées : la catégorie socioprofessionnelle des enfants, fortement corrélée au statut d'emploi de l'enfant et au niveau d'éducation du parent, et la proximité du lieu d'habitation des enfants par rapport à celui des parents, variable potentiellement endogène. Au lieu d'être un déterminant du comportement d'aide, la distance au parent peut en effet être expliquée par le comportement d'aide (cas de causalité inverse), mais aussi peut être une source potentielle de colinéarité : habiter hors de la région de son parent est fortement corrélé à la situation familiale et au statut d'emploi des enfants ainsi qu'au statut conjugal et au niveau d'études du parent ${ }^{(17)}$. Inclure cette variable peut donc non seule-

(16) Cela revient à croiser les deux indicatrices du sexe de chaque enfant. Le commentaire des résultats mentionne les résultats obtenus avec un modèle alternatif incluant deux indicatrices distinctes pour le sexe de chaque enfant sans effet croisé.

(17) Résultats obtenus par l'estimation d'un modèle probit expliquant la probabilité qu'un enfant habite hors de la région de son parent. 
ment biaiser les estimations mais aussi diminuer leur précision. Les tests de robustesse discutent toutefois des résultats d'estimations incluant ces deux variables explicatives.

\section{Résultats}

La description des configurations d'aide a montré que les cadets s'impliquent plus souvent que leurs aînés. Comme mentionné précédemment, cet écart peut s'expliquer par des caractéristiques individuelles différentes selon le rang. L'estimation du modèle montre qu'il est également dû à un impact différent des caractéristiques structurelles, surtout lorsqu'on mobilise une définition large de l'aide, et à l'existence d'interactions asymétriques.

\section{Différenciation des comportements selon le rang et le type d'aide}

Le tableau 6 présente les résultats des estimations du modèle ${ }^{(18)}$, avec deux définitions de l'aide. Dans la première estimation, nous avons étudié les déterminants de l'aide de l'aîné (colonne 1) et du cadet (colonne 2), en considérant comme aidants les enfants cohabitants ou déclarés aidants par le parent, quel que soit le type d'aide apportée. Dans la seconde estimation (colonnes 4 et 5), nous avons restreint le statut d'aidant aux enfants cohabitants ou déclarés aidants pour les tâches de la vie quotidienne.

Concernant l'effet des caractéristiques individuelles, des différences entre aînés et cadets apparaissent lorsqu'on utilise une définition large de l'aide. Limplication des cadets dépend significativement et négativement du fait d'être en couple, du fait d'avoir des enfants et du statut d'actif. L'implication des aînés, quant à elle, est affectée par leur âge et par le fait d'être en couple. Restreindre la définition de l'aide aux tâches de la vie quotidienne tend cependant à rapprocher les comportements des enfants. Le comportement de l'aîné devient sensible, comme pour les cadets, au fait d'avoir des enfants et au fait d'être actif ${ }^{(19)}$.

Concernant l'effet des caractéristiques des parents, les comportements entre aînés et cadets apparaissent similaires. Les enfants s'impliquent davantage quand le parent est âgé, qu'il vit sans conjoint et qu'il n'est pas diplômé du bac $^{(20)}$. Aînés et cadets ont également en commun d'être plus fréquemment

(18) Dans ces estimations, certains coefficients ont été contraints à être égaux : c'est ce que signalent les colonnes 3 et 6 . Le modèle a d'abord été estimé en autorisant l'ensemble des coefficients à être différents entre aîné et cadet. Dans un second temps, étant donnée la petite taille de notre échantillon - qui nous invite à être le plus parcimonieux possible dans le nombre de paramètres à estimer-, nous avons contraint les coefficients à être égaux entre aîné et cadet lorsqu'ils n'étaient pas significativement différents lors de la première estimation (au seuil de $20 \%$ ).

(19) On remarque également que les situations où le parent n'a pas pu renseigner le statut professionnel de l'enfant sont, dans tous les cas, associées à une moindre probabilité d'aide de l'enfant.

(20) Ce dernier effet pourrait être interprété comme une mesure indirecte du besoin d'aide du parent, en faisant l'hypothèse qu'un plus faible niveau d'études est associé à un moindre recours à l'aide professionnelle. 
impliqués quand le parent dépendant est leur mère, mais l'effet n'est significatif que pour la définition large de l'aide, suggérant que le sexe du parent dépendant importe plutôt pour l'aide financière ou le soutien moral. On retrouve cependant une divergence des comportements avec la définition large de l'aide. Le comportement des cadets se distingue en effet de celui des aînés à deux niveaux : ils aident d'autant plus souvent que le parent a une limitation ADL, quelle que soit la définition de l'aide; ils s'impliquent significativement moins quand le parent déclare un revenu mensuel supérieur à $1800 € /$ mois, mais là uniquement pour la définition large de l'aide, ce qui suggère que cette distinction concerne l'aide financière. Au-delà de la différence de comportements selon le rang, ces résultats renforcent l'impression, déjà perceptible pour les déterminants individuels, que cette différence plus nette dans le cas d'une définition large de l'aide tient à une relative rigidité du comportement des aînés.

Tableau 6. Déterminants de l'aide apportée à un parent âgé dépendant par les cadets et aînés des fratries de 2 enfants

\begin{tabular}{|c|c|c|c|c|c|c|}
\hline & \multicolumn{3}{|c|}{$\begin{array}{l}\text { Enfant déclaré aidant par le parent } \\
\text { (tous types d'aide) ou cohabitant }\end{array}$} & \multicolumn{3}{|c|}{$\begin{array}{c}\text { Enfant déclaré aidant par le parent } \\
\text { pour de l'aide aux tâches de la vie } \\
\text { quotidienne ou cohabitant }\end{array}$} \\
\hline & Aîné & Cadet & $\begin{array}{l}\text { Égalité des } \\
\text { coefficients }\end{array}$ & Aîné & Cadet & $\begin{array}{l}\text { Égalité des } \\
\text { coefficients }\end{array}$ \\
\hline Constante & $\begin{array}{r}-0,364 \\
(0,563)\end{array}$ & $\begin{array}{c}-0,364 \\
(0,563)\end{array}$ & Oui & $\begin{array}{r}-0,464 \\
(0,605)\end{array}$ & $\begin{array}{r}-0,464 \\
(0,605)\end{array}$ & Oui \\
\hline \multicolumn{7}{|l|}{ Caractéristiques de l'enfant } \\
\hline Âge & $\begin{array}{l}-0,036^{\star \star \star} \\
(0,011)\end{array}$ & $\begin{array}{r}-0,010 \\
(0,010)\end{array}$ & Non & $\begin{array}{l}-0,032^{* * *} \\
(0,011)\end{array}$ & $\begin{array}{r}-0,016 \\
(0,010)\end{array}$ & Non \\
\hline $\begin{array}{l}\text { En couple } \\
\quad \text { Réf. : n'est pas en couple }\end{array}$ & $\begin{array}{l}-0,492^{\star \star \star} \\
(0,124)\end{array}$ & $\begin{array}{l}-0,490^{* * *} \\
(0,124)\end{array}$ & Oui & $\begin{array}{l}-0,733^{* * *} \\
(0,126)\end{array}$ & $\begin{array}{l}-0,733^{\star \star *} \\
(0,126)\end{array}$ & Oui \\
\hline $\begin{array}{l}\text { A des enfants } \\
\text { Réf. : n'a pas d'enfants }\end{array}$ & $\begin{array}{r}-0,174 \\
(0,188)\end{array}$ & $\begin{array}{l}-0,550^{* * *} \\
(0,174)\end{array}$ & Non & $\begin{array}{l}-0,347 * * \\
(0,140)\end{array}$ & $\begin{array}{l}-0,347 * * \\
(0,140)\end{array}$ & Oui \\
\hline $\begin{array}{l}\text { Actif } \\
\text { (en emploi ou chômeur) }\end{array}$ & $\begin{array}{l}-0,177 \\
(0,158)\end{array}$ & $\begin{array}{l}-0,641^{* * *} \\
(0,158)\end{array}$ & Non & $\begin{array}{c}-0,308^{*} \\
(0,160)\end{array}$ & $\begin{array}{l}-0,823^{* \star *} \\
(0,159)\end{array}$ & Non \\
\hline $\begin{array}{l}\text { Le parent ne connaît pas } \\
\text { la profession de l'enfant } \\
\text { Réf. : retraité, étudiant, } \\
\text { au foyer ou autre }\end{array}$ & $\begin{array}{l}-1,434^{* * *} \\
(0,376)\end{array}$ & $\begin{array}{l}-1,1434^{* * *} \\
(0,376)\end{array}$ & Oui & $\begin{array}{l}-2,027 * * * \\
(0,469)\end{array}$ & $\begin{array}{l}-2,027^{* * *} \\
(0,469)\end{array}$ & Oui \\
\hline \multicolumn{7}{|l|}{ Caractéristiques du parent } \\
\hline Âge & $\begin{array}{l}0,026 * * * \\
(0,010)\end{array}$ & $\begin{array}{l}0,026^{* * *} \\
(0,010)\end{array}$ & Oui & $\begin{array}{l}0,026^{\star *} \\
(0,011)\end{array}$ & $\begin{array}{l}0,026^{* *} \\
(0,011)\end{array}$ & Oui \\
\hline $\begin{array}{l}\text { Femme } \\
\text { Réf. : homme }\end{array}$ & $\begin{array}{l}0,225^{* *} \\
(0,116)\end{array}$ & $\begin{array}{l}0,225^{* *} \\
(0,116)\end{array}$ & Oui & $\begin{array}{c}0,159 \\
(0,128)\end{array}$ & $\begin{array}{c}0,159 \\
(0,128)\end{array}$ & Oui \\
\hline $\begin{array}{l}\text { A des limitations ADL } \\
\text { Réf. : a uniquement des } \\
\text { limitations IADL }\end{array}$ & $\begin{array}{c}-0,073 \\
(0,165)\end{array}$ & $\begin{array}{l}0,368^{* *} \\
(0,151)\end{array}$ & Non & $\begin{array}{c}0,104 \\
(0,178)\end{array}$ & $\begin{array}{l}0,468^{\star * \star} \\
(0,157)\end{array}$ & Non \\
\hline $\begin{array}{l}\text { Diplômé du bac } \\
\text { Réf. : n'est pas diplômé } \\
\text { du bac }\end{array}$ & $\begin{array}{l}-0,519 * * * \\
(0,191)\end{array}$ & $\begin{array}{l}-0,519 * * * \\
(0,191)\end{array}$ & Oui & $\begin{array}{l}-0,707^{* * *} \\
(0,219)\end{array}$ & $\begin{array}{l}-0,707^{* * *} \\
(0,219)\end{array}$ & Oui \\
\hline $\begin{array}{l}\text { Revenu mensuel } \geq 1800 € \\
\text { Réf. : revenu mensuel } \\
<1800 €\end{array}$ & $\begin{array}{r}-0,116 \\
(0,159)\end{array}$ & $\begin{array}{l}-0,155^{* * *} \\
(0,149)\end{array}$ & Non & $\begin{array}{l}-0,123 \\
(0,114)\end{array}$ & $\begin{array}{c}-0,123 \\
(0,114)\end{array}$ & Oui \\
\hline $\begin{array}{l}\text { Vit sans conjoint } \\
\text { Réf. : vit avec un conjoint }\end{array}$ & $\begin{array}{l}0,394^{* * *} \\
(0,125)\end{array}$ & $\begin{array}{l}0,394^{* * *} \\
(0,125)\end{array}$ & Oui & $\begin{array}{l}0,271^{* *} \\
(0,124)\end{array}$ & $\begin{array}{l}0,271^{* *} \\
(0,124)\end{array}$ & Oui \\
\hline
\end{tabular}


Tableau 6 (suite). Déterminants de l'aide apportée à un parent âgé dépendant par les cadets et aînés des fratries de 2 enfants

\begin{tabular}{|c|c|c|c|c|c|c|}
\hline & \multicolumn{3}{|c|}{$\begin{array}{l}\text { Enfant déclaré aidant par le parent } \\
\text { (tous types d'aide) ou cohabitant }\end{array}$} & \multicolumn{3}{|c|}{$\begin{array}{l}\text { Enfant déclaré aidant par le parent } \\
\text { pour de l'aide aux tâches de la vie } \\
\text { quotidienne ou cohabitant }\end{array}$} \\
\hline & Aîné & Cadet & $\begin{array}{l}\text { Égalité des } \\
\text { coefficients }\end{array}$ & Aîné & Cadet & $\begin{array}{l}\text { Égalité des } \\
\text { coefficients }\end{array}$ \\
\hline \multicolumn{7}{|l|}{ Caractéristiques de la fratrie } \\
\hline $\begin{array}{l}\text { Autre enfant actif } \\
\text { Réf. : autre enfant retraité, } \\
\text { étudiant, au foyer, autre, } \\
\text { ou parent ne connaît pas } \\
\text { la profession de l'enfant }\end{array}$ & $\begin{array}{r}-0,132 \\
(0,129)\end{array}$ & $\begin{array}{r}-0,132 \\
(0,129)\end{array}$ & Oui & $\begin{array}{r}-0,076 \\
(0,138)\end{array}$ & $\begin{array}{c}-0,076 \\
(0,138)\end{array}$ & Oui \\
\hline $\begin{array}{l}\text { Ego }=\text { femme, } \\
\text { alter }=\text { femme } \\
\text { Réf. : ego = femme, } \\
\text { alter }=\text { homme }\end{array}$ & $\begin{array}{l}-0,399 * * \\
(0,175)\end{array}$ & $\begin{array}{r}-0,215 \\
(0,176)\end{array}$ & Non & $\begin{array}{c}-0,244^{\star} \\
(0,138)\end{array}$ & $\begin{array}{c}-0,244^{*} \\
(0,138)\end{array}$ & Oui \\
\hline $\begin{array}{l}\text { Ego }=\text { homme, } \\
\text { alter = homme } \\
\text { Réf. : ego = femme, } \\
\text { alter = homme }\end{array}$ & $\begin{array}{l}-0,459 * * * \\
(0,144)\end{array}$ & $\begin{array}{l}-0,459 * * * \\
(0,144)\end{array}$ & Oui & $\begin{array}{l}-0,481^{* * *} \\
(0,161)\end{array}$ & $\begin{array}{l}-0,481^{* * *} \\
(0,161)\end{array}$ & Oui \\
\hline $\begin{array}{l}\text { Ego }=\text { homme } \\
\text { alter }=\text { femme } \\
\text { Réf. : ego }=\text { femme, } \\
\text { alter }=\text { homme }\end{array}$ & $\begin{array}{l}-0,700 * * * \\
(0,190)\end{array}$ & $\begin{array}{c}-0,122 \\
(0,199)\end{array}$ & Non & $\begin{array}{l}-0,402^{\star * \star} \\
(0,145)\end{array}$ & $\begin{array}{l}-0,402^{* * *} \\
(0,145)\end{array}$ & Oui \\
\hline $\begin{array}{l}\text { Écart d'âge entre l'aîné } \\
\text { et le cadet }>10 \text { ans } \\
\text { Réf. : écart d'âge }<10 \text { ans }\end{array}$ & $\begin{array}{l}0,377 \\
(0,236)\end{array}$ & $\begin{array}{l}-0,720 * * * \\
(0,265)\end{array}$ & Non & $\begin{array}{l}0,433^{*} \\
(0,242)\end{array}$ & $\begin{array}{c}-0,514^{\star} \\
(0,280)\end{array}$ & Non \\
\hline \multicolumn{7}{|l|}{ Interactions } \\
\hline L'autre enfant est impliqué (a) & $\begin{array}{l}1,197^{* * *} \\
(0,360)\end{array}$ & $\begin{array}{c}-0,476 \\
(0,524)\end{array}$ & Non & $\begin{array}{l}1,004 * * * \\
(0,367)\end{array}$ & $\begin{array}{c}-0,373 \\
(0,428)\end{array}$ & Non \\
\hline $\begin{array}{l}\text { Coefficient de corrélation } \\
\text { entre les résidus }\end{array}$ & & $\begin{array}{l}0,090 \\
0,361)\end{array}$ & & & $\begin{array}{l}0,015 \\
0,322)\end{array}$ & \\
\hline Log vraisemblance & -48 & 6,795 & & -40 & 3,442 & \\
\hline Nombre d'observations & 47 & & & 47 & & \\
\hline \multicolumn{7}{|c|}{$\begin{array}{l}\text { (a) La fonction de vraisemblance n'étant pas différenciable au point où les coefficients d'interaction sont simul- } \\
\text { tanément égaux à } 0 \text {, il est nécessaire de mettre en œuvre un test de significativité spécifique, décrit formellement } \\
\text { dans Fontaine et al. (2009). } \\
\text { Significativité : }{ }^{*} p<0,10 ;{ }^{* *} p<0,05 ; * * * p<0,01 \text {. } \\
\text { Notes: Écarts types entre parenthèses. Dans l'estimation présentée dans les colonnes } 1 \text { et } 2 \text {, un enfant est aidant } \\
\text { s'il est déclaré aidant par le parent (tous types d'aide) ou s'il est cohabitant. Dans l'estimation présentée dans } \text { les colonnes } 4 \text { et } 5 \text {, un enfant est aidant s'il est déclaré aidant par le parent et apporte de l'aide aux tâches de } \\
\text { les colon } \\
\text { la vie quotidienne ou s'il est cohabitant. Les colonnes } 3 \text { et } 6 \text { montrent les coefficients contraints à être égaux } \\
\text { dans la procédure d'estimation, c'est-à-dire les coefficients pour lesquels la différence entre les coefficients de } \\
\text { l'aîné et du cadet dans le modèle sans contrainte n'était pas significative au seuil de } 20 \% \text {. } \\
\text { Champ : } 492 \text { personnes âgées et dépendantes vivant en ménage ordinaire et ayant } 2 \text { enfants majeurs. } \\
\text { Source : Enquête Handicap-Santé, volet Ménages (Insee-Drees, 2008). }\end{array}$} \\
\hline
\end{tabular}

Concernant les variables relatives à la fratrie, nos résultats soulignent, d'une manière inédite, la dimension relative de l'effet de genre. Ils montrent en effet que ce ne sont pas les femmes en général qui ont une probabilité plus forte d'être aidantes. Pour les aînées comme pour les cadettes dans le cas de la définition restreinte de l'aide, seules celles qui ont un frère sont plus fréquemment impliquées que tout autre enfant de même rang, toutes choses égales par ailleurs. Pour les cadettes, dans le cas de la définition large de l'aide, être une 
femme (plutôt qu'un homme) n'a d'effet que si l'aîné est un fils : autrement dit, quand l'aîné est une fille, cadets ou cadettes ont la même probabilité d'aider à autres caractéristiques données. Contrôler par le sexe au niveau individuel n'aurait pas permis de mettre en évidence cet effet qui existe conditionnellement au sexe de l'autre enfant ${ }^{(21)}$.

Par ailleurs, aînés et cadets ont également en commun d'être insensibles au statut d'actif du frère ou de la sœur, quelle que soit la définition de l'aide. En outre, un écart d'âge important entre l'aîné et le cadet réduit significativement la probabilité d'aide du cadet, quelle que soit la définition de l'aide; pour l'aîné, un tel écart d'âge augmente la probabilité d'être aidant dans le cas de la définition restreinte uniquement. Ce type de configuration pourrait renvoyer à des situations particulières où les aînés ont très tôt assumé un rôle d'aidant domestique (seconder leur parent, s'occuper du plus jeune) qu'ils perpétuent sous une forme nouvelle (aider leur parent, protéger leur cadet). Les aînés beaucoup plus âgés que leurs cadets pourraient également se conduire comme un enfant unique, ce qui les désigne systématiquement comme aidant (Weber, 2011).

Du point de vue des interactions endogènes, on observe enfin une asymétrie des comportements, que l'on restreigne l'aide aux tâches de la vie quotidienne ou que l'on élargisse le champ à l'aide financière et au soutien moral. Toutes choses égales par ailleurs, les aînés s'impliquent significativement plus fréquemment dans la prise en charge quand leur cadet est aidant; à l'inverse, les cadets tendraient à ne pas s'ajuster à l'implication de l'aîné, voire à s'impliquer moins fréquemment quand leur aîné est aidant (coefficient négatif, mais non significativement différent de zéro).

\section{Spécifications alternatives}

Afin de vérifier la robustesse des résultats qui viennent d'être présentés, plusieurs estimations alternatives ont été réalisées (pour la définition large de l'aide) ${ }^{(22)}$.

L'estimation d'un modèle intégrant la proximité géographique du lieu d'habitation des enfants par rapport à celui de leur parent, en distinguant les enfants habitant hors de la région du parent, rejoint les résultats classiques de la littérature (voir, par exemple, Mulder et van der Meer, 2009). Le fait d'habiter hors de la région diminue la probabilité d'être aidant. Les résultats de la section précédente sont robustes à l'inclusion de la variable de distance et le coefficient d'interaction endogène devient significatif pour le cadet ${ }^{(23)}$. Le modèle a été

(21) Dans un modèle avec deux indicatrices séparées pour le sexe de chaque enfant, être une femme augmente significativement la probabilité d'être aidante uniquement pour l'aîné, avec la définition large et la définition restreinte.

(22) Pour chaque spécification alternative, on a réitéré la procédure de sélection des contraintes d'égalité des coefficients.

(23) Le coefficient d'interaction du cadet, jusqu'ici non significatif, devient significativement différent de zéro au seuil de $5 \%$. 
également estimé avec une variable relative au lieu de vie du parent (« vit en milieu rural $»$ ou non). La résidence en milieu rural du parent n'affecte pas significativement la probabilité d'être aidant des enfants, et les résultats sur les autres déterminants sont robustes.

Nous avons ensuite inclus dans le modèle des indicatrices relatives aux catégories socioprofessionnelles des enfants, construites selon la nomenclature en six postes de l'Insee. Par rapport à la catégorie des cadres, le fait d'appartenir à la catégorie « ouvriers » ou " professions intermédiaires » pour l'aîné, " agriculteurs » pour le cadet, affecte positivement la probabilité d'être aidant. Pour faciliter l'interprétation, nous avons ensuite testé l'effet d'un écart dans les catégories socioprofessionnelles de l'aîné et du cadet, en différenciant les cas où l'un des enfants appartient à l'une des trois premières catégories de l'Insee (" agriculteurs ", « artisans, commerçants et chefs d'entreprises », « cadres et professions intellectuelles supérieures $»$ ), tandis que l'autre appartient aux trois dernières ( techniciens et autres professions intermédiaires », « employés », « ouvriers »). Dans le cas où l'aîné appartient aux premières catégories et le cadet à l'une des autres, la probabilité d'être aidant est plus faible pour le cadet comme pour l'aîné. Dans le cas inverse, il n'y a pas d'effet sur la probabilité d'aide des enfants.

D'autres variables ont été introduites, qui ne semblent pas modifier les équilibres familiaux observés jusqu'ici. Lorsque le parent ne vit pas en couple, un éventuel effet de "partage " pourrait affecter l'aide fournie par les enfants de parents divorcés ou séparés, notamment si le deuxième parent est également dépendant. Le modèle initial a donc été estimé avec une indicatrice relative au statut matrimonial du parent, distinguant les individus divorcés ou séparés des individus veufs ou célibataires. Cette estimation ne fait pas apparaître d'effet significatif de ces statuts matrimoniaux sur l'aide.

Enfin, pour prendre en compte l'aide formelle et l'aide informelle fournie par d'autres membres de l'entourage, nous avons estimé le modèle en incluant successivement une indicatrice qui distingue les individus recevant de l'aide professionnelle puis une autre indicatrice qui distingue les individus recevant de l'aide de la part d'un proche (membre de la famille hors conjoint ou enfants, ami, voisin). D’après ces estimations, les décisions d'implication des aînés et des cadets ne sont affectées ni par l'aide formelle ni par les autres aides informelles. Dans tous ces modèles, les résultats relatifs aux autres variables explicatives restent robustes à l'inclusion de ces indicatrices.

\section{Conclusion}

Nos résultats éclairent certains mécanismes guidant l'organisation familiale de la prise en charge d'un parent âgé dépendant. Ils confirment tout d'abord la sensibilité de l'implication des enfants aux configurations familiales. La présence d'un conjoint auprès du parent âgé dépendant réduit l'implication 
des enfants et module la sensibilité de l'implication des enfants à la taille de la fratrie. En outre, le fait d'être aidant dépend du rang dans la fratrie : ainsi, dans les fratries de 2 enfants, les cadets sont plus souvent déclarés aidants, et plus particulièrement en tant qu'aidant seul.

L'analyse toutes choses égales par ailleurs conduite sur les fratries de 2 enfants distingue plusieurs explications de ces différences. Tout d'abord, le fait d'aider dépend de caractéristiques individuelles qui sont réparties différemment parmi les aînés et les cadets. Ensuite, les caractéristiques déterminantes diffèrent selon le rang dans la fratrie. Le comportement des aînés apparaît moins sensible aux déterminants individuels que celui des cadets, qui semble renvoyer à des arbitrages entre coûts d'opportunité et bénéfices de l'aide : les cadets ajusteraient leur aide en fonction de leurs contraintes personnelles (statut familial, situation d'emploi) ou du niveau de besoin du parent. Quant aux aînés, ils semblent s'ajuster au comportement de leur cadet : ils sont d'autant plus fréquemment aidant que leur cadet aide, alors que l'ajustement des cadets est non significatif. Ces différences s'estompent cependant lorsqu'on s'intéresse aux seules aides pour les tâches de la vie quotidienne : dans ce cas, la différence essentielle entre aînés et cadets réside dans l'asymétrie des interactions endogènes, c'est-à-dire dans l'ajustement différentiel de l'un au comportement de l'autre. L'analyse révèle aussi l'importance de raisonner sur la composition sexuée des fratries : en effet, le fait d'être une femme (plutôt qu'un homme) n'apparaît significatif que lorsqu'on a un frère, de même que le fait d'avoir un frère (plutôt qu'une sœur) n'apparaît significatif que lorsqu'on est une femme.

Ces premiers résultats à partir de données françaises appellent des approfondissements. Tout d'abord, il faut noter la définition particulière du statut d'aidant reposant sur la déclaration du parent aidé. Cette définition n'est pas neutre et peut être à l'origine de biais déclaratifs dont il est difficile d'estimer le signe et l'ampleur. Il serait en outre opportun de clarifier les mécanismes sous-jacents aux différences de comportements d'aide selon le rang dans la fratrie : traduisent-elles des préférences individuelles face à l'aide filiale différentes selon le rang, ou des sollicitations ciblées de la part du parent? Des données sur les demandes exprimées ou la fréquence des contacts avec l'un ou l'autre membre de la fratrie seraient nécessaires à l'étude de cette question. On pourrait également prendre en compte l'ancienneté de la dépendance et de l'aide apportée par les enfants, pour observer une éventuelle différenciation des comportements selon l'historique de la configuration d'aide. Enfin, notre formalisation dichotomique des comportements d'aide (être aidant ou non) est relativement grossière. Elle ne dit rien des modalités de l'aide, notamment du volume en temps des aides apportées : une situation où les 2 enfants sont aidants peut renvoyer à des modes d'organisation et de répartition de l'aide très variés.

Nos résultats rejoignent ceux obtenus au niveau européen à partir de l'enquête SHARE (Fontaine et al., 2009), même si ceux-ci portaient sur un 
échantillon légèrement différent (parents vivant seuls uniquement) et apparaissent plus nuancés. Ces écarts pourraient s'expliquer par des différences dans le champ d'enquête (contexte français ou variété des contextes européens) ou dans la définition de l'aide adoptée (dans SHARE, la personne n'a pas à intervenir régulièrement ni en réponse à des restrictions d'activités pour être déclarée " aidante »). Toutefois, que ce soit au niveau français ou européen, l'asymétrie et le sens des interactions entre aînés et cadets sont similaires.

Remerciements : Les auteurs remercient Carole Bonnet pour la discussion de ce travail ainsi que Martin Chabert, Olivier Supplisson, Marianne Tenand et Florence Weber pour leurs relectures et nombreux commentaires. Les auteurs remercient le comité de rédaction de la revue Population et trois rapporteurs anonymes pour leurs retours, ainsi que les participants du séminaire «Économie des institutions » (SEI) du Centre d'économie de la Sorbonne (CES) et du séminaire interne du Bureau d'économie théorique et appliquée (BETA). 
ANNEXE 
Tableau A.1. Description de l'échantillon d'estimation (\%)

\begin{tabular}{|c|c|c|c|c|c|c|}
\hline & & $\begin{array}{c}\text { Toutes } \\
\text { configurations } \\
\text { (479 fratries) }\end{array}$ & $\begin{array}{c}\text { Aucun } \\
\text { n'est aidant } \\
\text { (250) }\end{array}$ & $\begin{array}{l}\text { Seul l'aîné } \\
\text { est aidant } \\
(60)\end{array}$ & $\begin{array}{l}\text { Seul le cadet } \\
\text { est aidant } \\
\text { (93) }\end{array}$ & $\begin{array}{l}\text { Les } 2 \text { enfants } \\
\text { sont aidants } \\
\text { (76) }\end{array}$ \\
\hline \multicolumn{7}{|c|}{ Caractéristiques de l'enfant } \\
\hline \multirow[t]{2}{*}{ Âge de l'enfant (ans) ${ }^{(a)}$} & Aîné & 52 & 50 & 53 & 55 & 53 \\
\hline & Cadet & 47 & 45 & 45 & 50 & 48 \\
\hline \multirow[t]{2}{*}{$\begin{array}{c}\text { Situation familiale : } \\
\text { en couple (\%) }\end{array}$} & Aîné & $\begin{array}{c}72 \\
(344)\end{array}$ & $\begin{array}{c}76 \\
(191)\end{array}$ & $\begin{array}{c}47 \\
(28)\end{array}$ & $\begin{array}{l}80 \\
(75)\end{array}$ & $\begin{array}{l}66 \\
(50)\end{array}$ \\
\hline & Cadet & $\begin{array}{c}69 \\
(329)\end{array}$ & $\begin{array}{c}77 \\
(193)\end{array}$ & $\begin{array}{c}62 \\
(37)\end{array}$ & $\begin{array}{l}57 \\
(53)\end{array}$ & $\begin{array}{l}60 \\
(46)\end{array}$ \\
\hline \multirow[t]{2}{*}{ a des enfants (\%) } & Aîné & $\begin{array}{c}80 \\
(385)\end{array}$ & $\begin{array}{c}82 \\
(204)\end{array}$ & $\begin{array}{c}70 \\
(42)\end{array}$ & $\begin{array}{l}82 \\
(76)\end{array}$ & $\begin{array}{l}83 \\
(63)\end{array}$ \\
\hline & Cadet & $\begin{array}{c}76 \\
(365)\end{array}$ & $\begin{array}{c}82 \\
(206)\end{array}$ & $\begin{array}{c}70 \\
(42)\end{array}$ & $\begin{array}{c}73 \\
(68)\end{array}$ & $\begin{array}{c}64 \\
(49)\end{array}$ \\
\hline \multirow{3}{*}{$\begin{array}{l}\text { Statut d'emploi : } \\
\text { actif (actif occupé } \\
\text { ou chômeur) (\%) }\end{array}$} & & & & & & \\
\hline & Aîné & $\begin{array}{c}68 \\
(323)\end{array}$ & $\begin{array}{c}76 \\
(205)\end{array}$ & $\begin{array}{c}51 \\
(28)\end{array}$ & $\begin{array}{l}55 \\
(49)\end{array}$ & $\begin{array}{l}61 \\
(41)\end{array}$ \\
\hline & Cadet & $\begin{array}{c}76 \\
(363)\end{array}$ & $\begin{array}{c}85 \\
(229)\end{array}$ & $\begin{array}{c}65 \\
(36)\end{array}$ & $\begin{array}{c}60 \\
(53)\end{array}$ & $\begin{array}{l}67 \\
(45)\end{array}$ \\
\hline \multirow[t]{2}{*}{$\begin{array}{l}\text { le parent ne sait pas } \\
(\%)\end{array}$} & Aîné & $\begin{array}{c}3 \\
(16)\end{array}$ & $\begin{array}{c}3 \\
(7)\end{array}$ & $\begin{array}{l}7 \\
(4)\end{array}$ & $\begin{array}{c}6 \\
(5)\end{array}$ & $\begin{array}{c}0 \\
(0)\end{array}$ \\
\hline & Cadet & $\begin{array}{c}2 \\
(10)\end{array}$ & $\begin{array}{c}3 \\
(7) \\
\end{array}$ & $\begin{array}{c}4 \\
(2)\end{array}$ & $\begin{array}{c}1 \\
(1)\end{array}$ & $\begin{array}{c}0 \\
(0)\end{array}$ \\
\hline \multicolumn{7}{|c|}{ Caractéristiques du parent } \\
\hline \multicolumn{2}{|l|}{ Âge du parent (ans) } & 77 & 75 & 80 & 80 & 79 \\
\hline \multicolumn{2}{|l|}{ Femme $(\%)$} & $\begin{array}{c}69 \\
(31)\end{array}$ & $\begin{array}{c}63 \\
(157)\end{array}$ & $\begin{array}{c}70 \\
(42)\end{array}$ & $\begin{array}{c}74 \\
(69)\end{array}$ & $\begin{array}{l}83 \\
(63)\end{array}$ \\
\hline \multicolumn{2}{|c|}{$\begin{array}{l}\text { Le parent déclare ne pas pouvoir } \\
\text { faire seul l'une des activités de la } \\
\text { vie quotidienne (ADL) (\%) }\end{array}$} & $\begin{array}{l}27 \\
(130)\end{array}$ & $\begin{array}{l}23 \\
(58)\end{array}$ & $\begin{array}{c}22 \\
(13)\end{array}$ & $\begin{array}{l}33 \\
(31)\end{array}$ & $\begin{array}{l}37 \\
(28)\end{array}$ \\
\hline \multicolumn{2}{|c|}{$\begin{array}{l}\text { Est diplômé au moins } \\
\text { du baccalauréat }(\%)\end{array}$} & $\begin{array}{c}11 \\
(54)\end{array}$ & $\begin{array}{c}16 \\
(40)\end{array}$ & $\begin{array}{l}3 \\
(2)\end{array}$ & $\begin{array}{l}8 \\
(7)\end{array}$ & $\begin{array}{c}7 \\
(5)\end{array}$ \\
\hline \multicolumn{2}{|c|}{ Revenu mensuel $\geq 1800 €(\%)$} & $\begin{array}{c}38 \\
(183)\end{array}$ & $\begin{array}{c}45 \\
(113) \\
\end{array}$ & $\begin{array}{c}43 \\
(26)\end{array}$ & $\begin{array}{l}33 \\
(31) \\
\end{array}$ & $\begin{array}{c}17 \\
(13) \\
\end{array}$ \\
\hline \multicolumn{7}{|c|}{ Caractéristiques de la fratrie } \\
\hline \multicolumn{2}{|c|}{ Aîné femme et cadet homme (\%) } & $\begin{array}{l}26 \\
(124)\end{array}$ & $\begin{array}{l}20 \\
(51)\end{array}$ & $\begin{array}{c}47 \\
(28)\end{array}$ & $\begin{array}{l}23 \\
(21)\end{array}$ & $\begin{array}{l}32 \\
(24)\end{array}$ \\
\hline \multicolumn{2}{|c|}{ Aîné homme et cadet femme (\%) } & $\begin{array}{l}27 \\
(130)\end{array}$ & $\begin{array}{c}27 \\
(68)\end{array}$ & $\begin{array}{l}15 \\
(9)\end{array}$ & $\begin{array}{c}38 \\
(35)\end{array}$ & $\begin{array}{c}24 \\
(18)\end{array}$ \\
\hline \multicolumn{2}{|c|}{ Aîné femme et cadet femme (\%) } & $\begin{array}{l}26 \\
(126)\end{array}$ & $\begin{array}{c}27 \\
(67)\end{array}$ & $\begin{array}{l}23 \\
(14)\end{array}$ & $\begin{array}{l}25 \\
(23)\end{array}$ & $\begin{array}{c}29 \\
(22)\end{array}$ \\
\hline \multicolumn{2}{|c|}{$\begin{array}{l}\text { Écart entre l'aîné et le cadet } \\
\geq 10 \text { ans (\%) }\end{array}$} & $\begin{array}{c}9 \\
(45) \\
\end{array}$ & $\begin{array}{c}7 \\
(18) \\
\end{array}$ & $\begin{array}{c}22 \\
(13) \\
\end{array}$ & $\begin{array}{c}9 \\
(8) \\
\end{array}$ & $\begin{array}{l}8 \\
(6) \\
\end{array}$ \\
\hline \multicolumn{7}{|c|}{$\begin{array}{l}\text { (a) La moyenne d'âge est calculée sur les enfants pour lesquels le parent a répondu (469 aînés, } 474 \text { cadets). } \\
\text { Pour l'estimation, on attribue aux valeurs manquantes l'âge moyen des aînés et des cadets. } \\
\text { Notes : les effectifs sont entre parenthèses. Les statistiques présentées ici sont les statistiques de l'échantillon } \\
\text { utilisé pour l'estimation du modèle et ne sont donc pas pondérées. Un enfant est considéré comme aidant s'il } \\
\text { est déclaré aidant par le parent (tous types d'aide) ou cohabitant. } \\
\text { Champ : } 479 \text { personnes âgées dépendantes vivant en ménage ordinaire et ayant } 2 \text { enfants majeurs. } \\
\text { Source : Enquête Handicap-Santé, volet Ménages (Insee-Drees, 2008). }\end{array}$} \\
\hline
\end{tabular}




\section{RÉFÉRENCES}

Beliard Aude, Billaud Solène, Perrin-Heredia Ana, Weber Florence, 2012, « Circulation de l'enquêteur, relations familiales et aide informelle : post-enquête qualitative sur les situations de handicap, la santé et l'aide aux personnes en situation de handicap », Drees-CNSA, 226 p.

Bonnet Carole, CAMBOIS Emmanuelle, CASES Chantal, GAYMU Joëlle, 2013, « La dépendance : quelles différences entre les hommes et les femmes? ", Gérontologie et société, 145(2), p. 55-66.

Byrne David, Goeree Michelle S., Hiedemann Bridget, Stern Steven, 2009, « Formal home health care, informal care, and family decision making », International Economic Review, 50(4), p. 1205-1242.

CHeCKOvich Tennille J., STERn Steven, 2002, « Shared caregiving responsibilities of adult siblings with elderly parents », The Journal of Human Resources, 37(3), p. 441-478.

Colombo Francesca, LlenA-NozAl Ana, Mercier Jérôme, TJAdENS Frits, 2011, Help Wanted? Providing and Paying for Long-Term Care, Paris, OECD Publishing, 336 p.

DÉSESQUelles Aline, BrouARD Nicolas, 2003, « Le réseau familial des personnes âgées de 60 ans ou plus vivant à domicile ou en institution », Population, 58(2), p. 201-227.

DUÉE Michel, REBILlARD Cyril, 2006, « La dépendance des personnes âgées : une projection en 2040 », Données sociales : La société française, 7, p. 613-619.

ENGERS Maxim, STERN Steven, 2002, "Long-term care and family bargaining ", International Economic Review, 43(1), p. 73-114.

FONTAINE Roméo, 2011, « Le soutien familial aux personnes âgées dépendantes. Analyses microéconométriques des comportements individuels et familiaux de prise en charge ", thèse de doctorat, Paris Dauphine, 244 p.

FOnTAine Roméo, GRAmain Agnès, WitTWER Jérôme, 2007, « Les configurations d'aide familiale mobilisées autour des personnes âgées dépendantes en Europe ", Économie et statistique, 403(1), p. 97-115.

Fontaine Roméo, Gramain Agnès, WitTWer Jérôme, 2009, « Providing care for an elderly parent: Interactions among siblings? », Health Economics, 18(9), p. 1011-1029.

HIEDEMANN Bridget, STERN Steven, 1999, « Strategic play among family members when making long-term care decisions », Journal of Economic Behavior E Organization, 40(1), p. 29-57.

LE BIHAN-Youinou Blanche, MARTIN Claude, 2006, « Travailler et prendre soin d'un parent âgé dépendant", Travail, genre et sociétés, 16(2), p. 77-96.

MANSKI Charles F., 2000, «Economic analysis of social interactions », The Journal of Economic Perspectives, 14(3), p. 115-136.

MASSON Luc, 2013, "Avez-vous eu des enfants? Si oui, combien? ", in Beffy M., Guedj H. (dir.), France, portrait social, Paris, Insee, p. 93-109.

Membrado Monique, 2013, « Le genre et le vieillissement : regard sur la littérature », Recherches féministes, 26(2), p. 5-24. 
Mulder Clara H., VAn Der MeER Marieke J., 2009, « Geographical distances and support from family members », Population, Space and Place, 15(4), p. 381-399.

OGG Jim, RENAUt Sylvie, TRABUt Loïc, 2015, « La corésidence familiale entre générations adultes : un soutien réciproque », Retraite et société, 1(70), p. 105-125.

PENNEC Simone, 2007, « Comment les enfants s'occupent-ils de leurs parents? Pratiques et logiques filiales », in Dreyer Pascal, Ennuyer Bernard (dir.), Quand nos parents vieillissent : prendre soin d'un parent âgé, Paris, Autrement, Mutations, p. 105-121.

PEYRACHE Malorie, OGG Jim, 2017, «Concilier vie professionnelle et aide informelle à un parent âgé. Un défi des 50-64 ans en Europe ", Revue européenne des sciences sociales, 55(1), p. 97-125.

PEZZIN Liliana E., STEINBERG SCHONE Barbara, 1999, « Intergenerational household formation, female labor supply and informal caregiving: A bargaining approach », The Journal of Human Resources, 34(3), p. 475-503.

PezZin Liliana E., POLLAK Robert A., STEINBERG SCHONE Barbara, 2007, « Efficiency in family bargaining: Living arrangements and caregiving decisions of adult children and disabled elderly parents », CESifo Economic Studies, 53(1), p. 69-96.

SloAn Frank A., PiCONE Gabriel, HoERGER Thomas J., 1997, « The supply of children's time to disabled elderly parents », Economic Inquiry, 35(2), p. 295-308.

SOUlLiER Noémie, 2012, "Aider un proche âgé à domicile : la charge ressentie ", Paris, Drees, Études et résultats, n 799, 8 p.

SOUllier Noémie, Weber Amandine, 2011, «L'implication de l'entourage et des professionnels auprès des personnes âgées à domicile », Paris, Drees, Études et résultats, $\mathrm{n}^{\circ} 771,8 \mathrm{p}$.

STERN Steven, 1994, "Two dynamic discrete choice estimation problems and simulation method solutions », The Review of Economics and Statistics, 76(4), p. 695-702.

TAMER Elie, 2003, « Incomplete simultaneous discrete response model with multiple equilibria », The Review of Economic Studies, 70(1), p. 147-165.

TENAND Marianne, 2016, «La barrière des 60 ans dans les dispositifs de compensation des incapacités : quels effets sur les aides reçues à domicile par les populations handicapée et dépendante? », Revue française des affaires sociales, 4, p. 129-155.

WEBER Florence, 2011, Handicap et dépendance : drames humains, enjeux politiques, Paris, Rue d'Ulm, Collection du Cepremap, 76 p.

Weber Florence, TRABUT Loïc, Billaud Solène (dir.), 2014, Le salaire de la confiance : l'aide à domicile aujourd'hui, Paris, Rue d'Ulm, 368 p. 


\section{Quitterie ROQUeberT, Roméo FontaIne, Agnès Gramain • AIDER UN PARENT ÂGÉ DÉPENDANT. CONFIGURATIONS D'AIDE ET INTERACTIONS DANS LES FRATRIES EN FRANCE}

À partir de l'enquête Handicap-Santé, volet Ménages (Insee-Drees, 2008), nous étudions les configurations d'aide familiale autour d'un parent âgé et dépendant en France. Une première étape descriptive montre que l'aide apportée par les enfants est affectée par le statut conjugal du parent, la taille de la fratrie et le rang dans la fratrie. L'analyse se concentre ensuite sur les familles de 2 enfants et montre que les écarts de mobilisation observés selon le rang découlent de trois sources : les différences de caractéristiques individuelles entre aînés et cadets, l'impact variable de ces caractéristiques sur la décision d'aide, et enfin une différence dans l'ajustement des uns au comportement des autres (interactions endogènes). L'impact des caractéristiques familiales apparaît en effet relativement similaire pour les aînés et les cadets -en particulier la forte assignation au rôle d'aidant des femmes avec un frère-, mais le comportement des cadets fait apparaître en outre un arbitrage entre coûts et utilité de l'aide. Avec une définition de l'aide ciblée sur les tâches de la vie quotidienne, une seule explication majeure des différences demeure : I'asymétrie dans l'ajustement au comportement de I'autre enfant, à caractéristiques individuelles et familiales données.

\section{Quitterie Roquebert, Roméo FontaIne, Agnès Gramain • CARING FOR A DePendent Elderly Parent: CARe ARRANGements and Sibling InTERACTIONS IN France}

Using data from the Household section of the Handicap-Santé (Disability and health) survey, (INSEE-DREES, 2008), this article studies kin support arrangements for dependent older adults in France. The first part is descriptive, showing how the care provided by adult children is affected by the parent's conjugal situation, sibship size and birth order. The second part analyses families with two adult children and shows that the observed differences in care involvement by birth order are due to three factors: differences in individual characteristics between elder and younger siblings, differences in the impacts of these characteristics on the caregiving decision, and a difference in the adjustment of one sibling to the behaviour of the other (endogenous interactions). The impact of family characteristics seems fairly similar for elder and younger siblings, especially the strong tendency for the woman to be the caregiver if her sibling is a man, but the behaviour of the younger children reflects a compromise between the costs and benefits of caregiving. Defining aid more narrowly as help with daily life tasks, just one major explanation of the differences remains: with a given set of individual and family characteristics, there is an asymmetry in adjustment to the caregiving behaviour of the other child.

\section{Quitterie ROQUeberT, Roméo FontaInE, Agnès GRAMAIN • AYUdAR A LOS PADRES MAYORES DEPENDIENTES. CONFIGURACIONES DE LA AYUDA E INTERACCIONES ENTRE LOS HIJOS EN FRANCIA}

A partir de la encuesta Handicap-Santé, en su componente Ménages (Insee-Drees, 2008), analizamos las configuraciones que presenta en Francia la ayuda familiar a los padres mayores dependientes. Una primera etapa descriptiva muestra que la ayuda aportada por los hijos está influida por el estatuto matrimonial del que recibe la ayuda, el número de hijos y el rango en la hermandad. El análisis se concentra después en las familias de dos hijos y se observa entonces que las diferencias de movilización según el rango resultan de tres fuentes: las diferencias en las características individuales entre primogénitos y cadetes, el impacto diferente de estas características sobre la decisión de ayudar, y en fin una diferencia en el ajuste de unos al comportamiento de los otros (interacciones endógenas). El impacto de las características familiares aparece en efecto relativamente similar en los primogénitos y en los cadetes - en particular la fuerte asignación de la hija al papel de ayudante cuando el otro hijo es un varón -, pero el comportamiento de los cadetes revela además un arbitraje entre coste y utilidad de la ayuda. Cuando se restringe la definición de ayuda a las tareas domésticas de la vida cotidiana, una sola explicación principal de las diferencias aparece: la asimetría en el ajustamiento al comportamiento del otro hijo, una vez fijadas las características individuales y familiares.

Mots-clés : personnes âgées dépendantes, aide informelle, interactions sociales, enquête Handicap-Santé, SHARE, France

Keywords: dependent older adults, informal care, social interactions, Handicap-Santé disability survey, SHARE, France 
